ANNALES

POLONICI MATHEMATICI

LXXIX.1 (2002)

\title{
On the class of functions defined in a halfplane and starlike with respect to the boundary point
}

\author{
by ADAm LECKO (Rzeszów)
}

\begin{abstract}
The purpose of this paper is to study the class $\mathcal{S}_{\infty}^{*}(\mathbb{H})$ of univalent analytic functions defined in the right halfplane $\mathbb{H}$ and starlike w.r.t. the boundary point at infinity. An analytic characterization of functions in $\mathcal{S}_{\infty}^{*}(\mathbb{H})$ is presented.
\end{abstract}

1. Introduction. The aim of this paper is to study the class $\mathcal{S}_{\infty}^{*}(\mathbb{H})$ of univalent analytic functions defined in the right halfplane $\mathbb{H}$ and starlike w.r.t. the boundary point at infinity.

The geometric definition of functions in $\mathcal{S}_{\infty}^{*}(\mathbb{H})$ is clear: together with every point $w \in f(\mathbb{H})$, the radial halfline with endpoint at $w$ lies in $f(\mathbb{H})$. This property was considered in $[4,6]$ where properties of functions in $\mathcal{S}_{\infty}^{*}(\mathbb{H})$ with the additional (so called) hydrodynamic normalization and a generalization were examined.

In this paper, developing another method based on the Julia Lemma reformulated in $\mathbb{H}$, we are able to improve the results of [6] considerably. The main results are presented in Theorems 3.1-3.3. Instead of the class $P$ used in [6] of functions having positive real part in $\mathbb{H}$ and hydrodynamically normalized we introduce the class $\mathcal{P}(\alpha ; \mathbb{H})$ of functions also having positive real part in $\mathbb{H}$ but with the boundary property which follows from the Julia Lemma. It is shown that $\mathcal{P}(\alpha ; \mathbb{H})$ is essentially larger than $P$. The key geometric property of functions in $\mathcal{S}_{\infty}^{*}(\mathbb{H})$ is preserving starlikeness in every halfplane contained in $\mathbb{H}$; this is proved in Theorem 3.1. Having this inner geometric behaviour we are able to find an analytic characterization of the class $\mathcal{S}_{\infty}^{*}(\mathbb{H})$ in terms of the corresponding class $\mathcal{P}(\alpha ; \mathbb{H})$; this is the subject of Theorems 3.2 and 3.3 .

Examples of functions show usefulness of these results.

2000 Mathematics Subject Classification: Primary 30C45.

Key words and phrases: analytic functions in a halfplane, functions starlike with respect to the boundary point, Julia Lemma, geometric characterization. 
2. The Julia Lemmas. Let $\overline{\mathbb{C}}=\mathbb{C} \cup\{\infty\}, \mathbb{D}=\{z \in \mathbb{C}:|z|<1\}$ and $\mathbb{T}=\partial \mathbb{D}$. For each $k>0$, we define the oricycle

$$
\mathbb{O}_{k}=\left\{z \in \mathbb{D}: \frac{|1-z|^{2}}{1-|z|^{2}}<k\right\} .
$$

It is a disk in $\mathbb{D}$ whose boundary circle $\partial \mathbb{O}_{k}$ is tangent to $\mathbb{T}$ at $z=1$. The center of $\mathbb{O}_{k}$ is at $(1 /(1+k), 0)$ and its radius is $k /(k+1)$.

For $\xi \in \mathbb{D}$ define the Möbius transformation

$$
\varphi_{\xi}(z)=\frac{z-\xi}{1-\bar{\xi} z}, \quad z \in \mathbb{C} \backslash\{1 / \bar{\xi}\} .
$$

For every $\xi \in \mathbb{D}$ the function $\varphi_{\xi}$ is an analytic automorphism of $\mathbb{D}$. The set of all analytic automorphisms of $\mathbb{D}$ will be denoted by $\operatorname{Aut}(\mathbb{D})$. It is well known that $f \in \operatorname{Aut}(\mathbb{D})$ iff there exist $\xi \in \mathbb{D}$ and $\lambda \in \mathbb{T}$ such that $f(z)=$ $\lambda \varphi_{\xi}(z), z \in \mathbb{D}$, i.e. every analytic automorphism of $\mathbb{D}$ is the composition of a rotation and a Möbius transformation.

The set of all analytic functions $\omega$ in $\mathbb{D}$ such that $|\omega(z)|<1$ for $z \in \mathbb{D}$ will be denoted by $\mathcal{B}$.

The following lemma ([3]; see also $[1$, p. 56]) is the basis for our considerations.

Lemma 2.1 (Julia). Let $\omega \in \mathcal{B}$. Assume that there exists a sequence $\left(z_{n}\right)$ of points in $\mathbb{D}$ such that

$$
\begin{gathered}
\lim _{n \rightarrow \infty} z_{n}=1, \quad \lim _{n \rightarrow \infty} \omega\left(z_{n}\right)=1, \\
\lim _{n \rightarrow \infty} \frac{1-\left|\omega\left(z_{n}\right)\right|}{1-\left|z_{n}\right|}=\alpha<\infty .
\end{gathered}
$$

Then

$$
\frac{|1-\omega(z)|^{2}}{1-|\omega(z)|^{2}} \leq \alpha \frac{|1-z|^{2}}{1-|z|^{2}}, \quad z \in \mathbb{D}
$$

and hence, for every $k>0$,

$$
\omega\left(\mathbb{O}_{k}\right) \subset \mathbb{O}_{\alpha k}
$$

Equality in (2.3) for some $z \in \mathbb{D}$ can occur only for $\omega \in \operatorname{Aut}(\mathbb{D})$, i.e., for $\omega(z)=\lambda \varphi_{\xi}(z), \quad z \in \mathbb{D}$, where $\xi \in \partial \mathbb{O}_{1 / \alpha}$ and $\lambda=(1-\bar{\xi}) /(1-\xi)$. In particular, equality holds for $\xi=(\alpha-1) /(\alpha+1)$ and $\lambda=1$.

REMARK 2.1. Since

$$
\frac{1-|\omega(z)|}{1-|z|} \geq \frac{1-|\omega(0)|}{1+|\omega(0)|}, \quad z \in \mathbb{D},
$$

for every $\omega \in \mathcal{B}$, the constant $\alpha$ defined in (2.2) is positive (see [1, p. 43]).

The Julia Lemma can be easily reformulated for the right halfplane. First we introduce some notations. Let $\mathbb{H}_{c}=\{z \in \mathbb{C}: \operatorname{Re} z>c\}, c \geq 0$, and 
$\mathbb{H}=\mathbb{H}_{0}$. Let $\mathcal{A}(\mathbb{H})$ denote the set of all analytic functions in $\mathbb{H}$ and let

$$
\mathcal{P}(\mathbb{H})=\{q \in \mathcal{A}(\mathbb{H}): \operatorname{Re} q(w)>0, w \in \mathbb{H}\} .
$$

Lemma 2.2. Let $q \in \mathcal{P}(\mathbb{H})$. Assume that there exists a sequence $\left(w_{n}\right)$ of points in $\mathbb{H}$ such that

$$
\begin{gathered}
\lim _{n \rightarrow \infty} w_{n}=\infty, \quad \lim _{n \rightarrow \infty} q\left(w_{n}\right)=\infty, \\
\lim _{n \rightarrow \infty}\left\{\frac{\left|q\left(w_{n}\right)+1\right|-\left|q\left(w_{n}\right)-1\right|}{\left|w_{n}+1\right|-\left|w_{n}-1\right|} \cdot \frac{\left|w_{n}+1\right|}{\left|q\left(w_{n}\right)+1\right|}\right\}=\alpha<\infty .
\end{gathered}
$$

Then

$$
\operatorname{Re} q(w) \geq \frac{1}{\alpha} \operatorname{Re} w, \quad w \in \mathbb{H},
$$

and hence, for every $c>0$,

$$
q\left(\mathbb{H}_{c}\right) \subset \mathbb{H}_{c / \alpha} .
$$

The result is sharp. Equality in (2.6) can occur only for

$$
q(w)=\frac{1}{\alpha} w+i t, \quad w \in \mathbb{H}, t \in \mathbb{R} .
$$

In particular, equality holds for

$$
q(w)=\frac{1}{\alpha} w, \quad w \in \mathbb{H} .
$$

Proof. Define

$$
\omega(z)=\frac{q\left(\frac{1+z}{1-z}\right)-1}{q\left(\frac{1+z}{1-z}\right)+1}, \quad z \in \mathbb{D} .
$$

For the sequence $\left(w_{n}\right)$ of points in $\mathbb{H}$ let

$$
z_{n}=\frac{w_{n}-1}{w_{n}+1}, \quad n \in \mathbb{N},
$$

be the corresponding sequence of points in $\mathbb{D}$. Since $\lim _{n \rightarrow \infty} w_{n}=\infty$, we have $\lim _{n \rightarrow \infty} z_{n}=1$. From (2.4) it follows that

$$
\lim _{n \rightarrow \infty} \omega\left(z_{n}\right)=\lim _{n \rightarrow \infty} \frac{q\left(w_{n}\right)-1}{q\left(w_{n}\right)+1}=1
$$

so (2.1) holds. In view of (2.5) and (2.8) we see that 


$$
\begin{aligned}
& \lim _{n \rightarrow \infty} \frac{1-\left|\omega\left(z_{n}\right)\right|}{1-\left|z_{n}\right|}=\lim _{n \rightarrow \infty} \frac{1-\left|\frac{q\left(w_{n}\right)-1}{q\left(w_{n}\right)+1}\right|}{1-\left|\frac{w_{n}-1}{w_{n}+1}\right|} \\
& \quad=\lim _{n \rightarrow \infty}\left\{\frac{\left|q\left(w_{n}\right)+1\right|-\left|q\left(w_{n}\right)-1\right|}{\left|w_{n}+1\right|-\left|w_{n}-1\right|} \cdot \frac{\left|w_{n}+1\right|}{\left|q\left(w_{n}\right)+1\right|}\right\}=\alpha<\infty .
\end{aligned}
$$

Hence (2.2) holds. On the other hand, by (2.8) we have

$$
q(w)=\frac{1+\omega(z)}{1-\omega(z)},
$$

where $w=(1+z) /(1-z), z \in \mathbb{D}$, so

$$
\operatorname{Re} q(w)=\frac{1-|\omega(z)|^{2}}{|1-\omega(z)|^{2}}, \quad \operatorname{Re} w=\frac{1-|z|^{2}}{|1-z|^{2}} .
$$

Hence (2.3) yields the assertion.

Suppose now that $q\left(w_{0}\right) \in \partial \mathbb{H}_{c / \alpha}$ for some $w_{0} \in \partial \mathbb{H}_{c}$ and $c>0$. Then $z_{0}=\left(w_{0}-1\right) /\left(w_{0}+1\right) \in \mathbb{D}$ lies on the oricycle $\partial \mathbb{O}_{1 / c}$ and $\omega\left(z_{0}\right) \in \partial \mathbb{O}_{\alpha / c}$. By the Julia Lemma this holds only for $\omega(z)=\lambda \varphi_{\xi}(z), z \in \mathbb{D}$, where $\xi \in \partial \mathbb{O}_{1 / \alpha}$ and $\lambda=(1-\bar{\xi}) /(1-\xi)$. Using (2.10) we find that

$$
q(w)=\frac{1+\lambda \varphi_{\xi}(z)}{1-\lambda \varphi_{\xi}(z)}=\frac{|1-\xi|^{2}}{1-|\xi|^{2}} w-i \frac{2 \operatorname{Im} \xi}{1-|\xi|^{2}}=\frac{1}{\alpha} w+i t,
$$

where $t \in \mathbb{R}$.

In particular, $t=0$ for $\xi=(\alpha-1) /(\alpha+1)$ and $\lambda=1$, which yields $q(w)=w / \alpha$.

Remark 2.2. By Remark 2.1 and (2.9), $\alpha$ defined in (2.5) is positive.

Suppose now that the sequence $\left(w_{n}\right)$ consists of only positive real numbers. Then we can formulate the following corollary.

Corollary 2.1. Let $q \in \mathcal{P}(\mathbb{H})$. Assume that there exists a sequence $\left(x_{n}\right)$ of positive real numbers such that

$$
\begin{gathered}
\lim _{n \rightarrow \infty} x_{n}=\infty, \quad \lim _{n \rightarrow \infty} q\left(x_{n}\right)=\infty, \\
\lim _{n \rightarrow \infty}\left\{\left(\left|q\left(x_{n}\right)+1\right|-\left|q\left(x_{n}\right)-1\right|\right) \frac{x_{n}+1}{\left|q\left(x_{n}\right)+1\right|}\right\}=2 \alpha<\infty .
\end{gathered}
$$

Then $q\left(\mathbb{H}_{c}\right) \subset \mathbb{H}_{c / \alpha}$ for every $c>0$. The result is sharp and (2.7) is the extremal function.

If also the sequence $\left(q\left(x_{n}\right)\right)$ consists of only positive real numbers then we have

Corollary 2.2. Let $q \in \mathcal{P}(\mathbb{H})$. Assume that there exists a sequence $\left(x_{n}\right)$ of positive real numbers such that $q\left(x_{n}\right)$ is a positive real number for 
every $n \in \mathbb{N}, \lim _{n \rightarrow \infty} x_{n}=\infty$ and

$$
\lim _{n \rightarrow \infty} \frac{q\left(x_{n}\right)}{x_{n}}=\frac{1}{\alpha}<\infty .
$$

Then $q\left(\mathbb{H}_{c}\right) \subset \mathbb{H}_{c / \alpha}$ for every $c>0$. The result is sharp and (2.7) is the extremal function.

Since $\left(x_{n}\right)$ tends to infinity, by (2.13) the same holds for $\left(q\left(x_{n}\right)\right)$. By Remark $2.2, \alpha>0$.

It is possible to consider stronger assumptions instead of (2.4). We can set

$$
\lim _{n \rightarrow \infty} \frac{q\left(w_{n}\right)}{w_{n}}=\beta
$$

with the sequence $\left(w_{n}\right)$ tending to infinity, which implies that $\lim _{n \rightarrow \infty} q\left(w_{n}\right)$ $=\infty$. Then we have

Corollary 2.3. Let $q \in \mathcal{P}(\mathbb{H})$. Assume that there exists a sequence $\left(w_{n}\right)$ of points in $\mathbb{H}$ such that

$$
\begin{gathered}
\lim _{n \rightarrow \infty} w_{n}=\infty, \quad \lim _{n \rightarrow \infty} \frac{q\left(w_{n}\right)}{w_{n}}=\beta \neq 0, \\
\frac{1}{|\beta|} \lim _{n \rightarrow \infty}\left\{\frac{\left|q\left(w_{n}\right)+1\right|-\left|q\left(w_{n}\right)-1\right|}{\left|w_{n}+1\right|-\left|w_{n}-1\right|}\right\}=\alpha<\infty .
\end{gathered}
$$

Then $q\left(\mathbb{H}_{c}\right) \subset \mathbb{H}_{c / \alpha}$ for every $c>0$. The result is sharp and (2.7) is the extremal function.

Suppose again that the sequence $\left(w_{n}\right)$ consists of only positive real numbers. Then we have

Corollary 2.4. Let $q \in \mathcal{P}(\mathbb{H})$. Assume that there exists a sequence $\left(x_{n}\right)$ of positive real numbers such that

$$
\lim _{n \rightarrow \infty} x_{n}=\infty, \quad \lim _{n \rightarrow \infty} \frac{q\left(x_{n}\right)}{x_{n}}=\beta \in \mathbb{C} \backslash\{0\}
$$

and

$$
\frac{1}{|\beta|} \lim _{n \rightarrow \infty}\left\{\left|q\left(x_{n}\right)+1\right|-\left|q\left(x_{n}\right)-1\right|\right\}=2 \alpha<\infty .
$$

Then $q\left(\mathbb{H}_{c}\right) \subset \mathbb{H}_{c / \alpha}$ for every $c>0$. The result is sharp and (2.7) is the extremal function.

A special case of Lemma 2.2 was proved in [6]. Let us recall this result.

TheOREM $2.1([6])$. Let $q \in \mathcal{P}(\mathbb{H})$. If the following limit exists:

$$
\lim _{w \rightarrow \infty}(q(w)-w)=a \neq \infty
$$

then $q\left(\mathbb{H}_{c}\right) \subset \mathbb{H}_{c}$ for every $c>0$. 
REMARK 2.3. The normalization (2.15) generalizes the so called hydrodynamic normalization $(a=0)$. Notice that $\operatorname{Re} a \geq 0$. The method of proof of Theorem 2.1 was based on the maximum principle for harmonic functions.

Now we show that Theorem 2.1 is a special case of Lemma 2.2. In fact we will prove that Theorem 2.1 is a consequence of Corollary 2.1. From (2.15) it follows that

$$
\begin{gathered}
\lim _{w \rightarrow \infty} q(w)=\infty \\
\lim _{w \rightarrow \infty} \operatorname{Re}(q(w)-w)=\operatorname{Re} a, \quad \lim _{w \rightarrow \infty} \operatorname{Im}(q(w)-w)=\operatorname{Im} a .
\end{gathered}
$$

Since by (2.15),

$$
\lim _{w \rightarrow \infty}(q(w)-w)=\lim _{w \rightarrow \infty} w\left(\frac{q(w)}{w}-1\right)=a,
$$

we have

$$
\lim _{w \rightarrow \infty} \frac{q(w)}{w}=1 .
$$

By (2.15) there exists a sequence $\left(x_{n}\right)$ of positive real numbers such that $\lim _{n \rightarrow \infty} x_{n}=\infty$ and $\lim _{n \rightarrow \infty}\left(q\left(x_{n}\right)-x_{n}\right)=a$. By (2.16), $\lim _{n \rightarrow \infty} q\left(x_{n}\right)$ $=\infty$ so $(2.11)$ is satisfied. Writing $q\left(x_{n}\right)=u_{n}+i v_{n}$, by (2.17) we have $\lim _{n \rightarrow \infty}\left(u_{n}-x_{n}\right)=\operatorname{Re} a$ and consequently,

$$
\lim _{n \rightarrow \infty} u_{n}=\infty \text {. }
$$

Also

$$
\lim _{n \rightarrow \infty} v_{n}=\operatorname{Im} a
$$

from (2.17). Since by (2.19) and (2.20),

$$
\begin{aligned}
\lim _{n \rightarrow \infty}\left|1+\frac{1}{q\left(x_{n}\right)}\right|^{2} & =\lim _{n \rightarrow \infty} \frac{\left(u_{n}+1\right)^{2}+v_{n}^{2}}{u_{n}^{2}+v_{n}^{2}} \\
& =\lim _{n \rightarrow \infty} \frac{\left(1+\frac{1}{u_{n}}\right)^{2}+\left(\frac{v_{n}}{u_{n}}\right)^{2}}{1+\left(\frac{v_{n}}{u_{n}}\right)^{2}}=1,
\end{aligned}
$$

(2.18) yields

$$
\lim _{n \rightarrow \infty} \frac{x_{n}+1}{\left|q\left(x_{n}\right)+1\right|}=\lim _{n \rightarrow \infty} \frac{x_{n}\left(1+\frac{1}{x_{n}}\right)}{\left|q\left(x_{n}\right)\right|\left|1+\frac{1}{q\left(x_{n}\right)}\right|}=1 .
$$


Using (2.19) and (2.20) we obtain

$$
\begin{aligned}
& \lim _{n \rightarrow \infty}\left\{\left|q\left(x_{n}\right)+1\right|-\left|q\left(x_{n}\right)-1\right|\right\} \\
= & \lim _{n \rightarrow \infty}\left\{\sqrt{\left(u_{n}+1\right)^{2}+v_{n}^{2}}-\sqrt{\left(u_{n}-1\right)^{2}+v_{n}^{2}}\right\} \\
= & \lim _{n \rightarrow \infty}\left\{\frac{4 u_{n}}{\left.\sqrt{\left(u_{n}+1\right)^{2}+v_{n}^{2}}+\sqrt{\left(u_{n}-1\right)^{2}+v_{n}^{2}}\right\}}\right. \\
= & \lim _{n \rightarrow \infty}\left\{\frac{4}{\sqrt{\left(1+\frac{1}{u_{n}}\right)^{2}+\left(\frac{v_{n}}{u_{n}}\right)^{2}}+\sqrt{\left(1-\frac{1}{u_{n}}\right)^{2}+\left(\frac{v_{n}}{u_{n}}\right)^{2}}}\right\}=2 .
\end{aligned}
$$

This result together with (2.21) yields (2.12) for $\alpha=1$. Hence the conclusion of the theorem follows.

EXAMPLES. 1. In fact, the conditions (2.4) and (2.5) are more general than (2.15). As an example, take

$$
q(w)=i \log w+w+\pi / 2, \quad w \in \mathbb{H} .
$$

It is easy to verify that $\operatorname{Re} q(w)>0$ for $w \in \mathbb{H}$.

Let $\left(x_{n}\right)$ be a sequence of positive real numbers such that $\lim _{n \rightarrow \infty} x_{n}=\infty$. Then

$$
\lim _{n \rightarrow \infty} q\left(x_{n}\right)=\lim _{n \rightarrow \infty}\left\{x_{n}+\pi / 2+i \log x_{n}\right\}=\infty
$$

so $(2.11)$ is true.

Repeating exactly calculations (2.21) and (2.22) with $u_{n}=x_{n}+\pi / 2$, $v_{n}=\log x_{n}$ and using the elementary fact that $\lim _{n \rightarrow \infty}\left(\log x_{n}\right) / x_{n}=0$ we infer that the limit (2.12) exists with $\alpha=1$.

On the other hand

$$
\lim _{w \rightarrow \infty}(q(w)-w)=\lim _{w \rightarrow \infty}(i \log w+\pi / 2)=\infty
$$

so (2.15) does not hold.

2. Every function $q \in \mathcal{P}(\mathbb{H})$ of the form

$$
q(w)=\beta w+o(w), \quad w \in \mathbb{H},
$$

with $\beta>0$ satisfies the assumptions of Corollary 2.4. Let $\left(x_{n}\right)$ be a sequence of positive real numbers such that $\lim _{n \rightarrow \infty} x_{n}=\infty$. Then

$$
\lim _{n \rightarrow \infty} \frac{q\left(x_{n}\right)}{x_{n}}=\beta+\lim _{n \rightarrow \infty} \frac{o\left(x_{n}\right)}{x_{n}}=\beta .
$$


Let $o\left(x_{n}\right)=s_{n}+i v_{n}$ and $q\left(x_{n}\right)=\beta x_{n}+s_{n}+i v_{n}$ for $n \in \mathbb{N}$. Since

$$
\lim _{n \rightarrow \infty} \frac{o\left(x_{n}\right)}{x_{n}}=\lim _{n \rightarrow \infty}\left(\frac{s_{n}}{x_{n}}+i \frac{v_{n}}{x_{n}}\right)=0
$$

we have

$$
\lim _{n \rightarrow \infty} \frac{s_{n}}{x_{n}}=0, \quad \lim _{n \rightarrow \infty} \frac{v_{n}}{x_{n}}=0 .
$$

Repeating the calculations in (2.21) and (2.22) with $u_{n}=\beta x_{n}+s_{n}$ and $v_{n}$ we obtain

$$
\lim _{n \rightarrow \infty} \frac{x_{n}+1}{\left|q\left(x_{n}\right)+1\right|}=\frac{1}{\beta}
$$

and

$$
\begin{aligned}
& \lim _{n \rightarrow \infty}\left\{\left|q\left(x_{n}\right)+1\right|-\left|q\left(x_{n}\right)-1\right|\right\} \\
& =\lim _{n \rightarrow \infty}\left\{\frac{4}{\sqrt{\left(1+\frac{1}{\beta x_{n}+s_{n}}\right)^{2}+\left(\frac{v_{n}}{\beta x_{n}+s_{n}}\right)^{2}}+\sqrt{\left(1-\frac{1}{\beta x_{n}+s_{n}}\right)^{2}+\left(\frac{v_{n}}{\beta x_{n}+s_{n}}\right)^{2}}}\right\}=2
\end{aligned}
$$

since

$$
\lim _{n \rightarrow \infty} \frac{v_{n}}{\beta x_{n}+s_{n}}=\lim _{n \rightarrow \infty} \frac{v_{n} / x_{n}}{\beta+s_{n} / x_{n}}=0 .
$$

Hence (2.12) holds with $\alpha=1 / \beta$.

The class of functions of the form (2.23) is essentially larger than this normalized by (2.15). The function $q(w)=\beta w+w^{\mu}, w \in \mathbb{H}$, for $\beta>0$ and $\mu \in[0,1)$ is of the form $(2.23)$.

3. Starlike functions defined in a halfplane. Classical geometric properties like starlikeness and convexity of analytic functions in the right halfplane were studied in $[2,6]$.

For $v \in \mathbb{C}$, let $l^{+}[v]=\{s v: s \in[1,+\infty)\}$ be the radial halfline with endpoint at $v$.

Let us start with the following definitions.

Definition 3.1. A simply connected domain $\Omega \subset \mathbb{C}, \Omega \neq \mathbb{C}$, with $\infty \in \partial \Omega$ is called starlike with respect to the boundary point at infinity if the halfline $l^{+}[v]$ is contained in $\Omega$ for every $v \in \Omega$. The set of all such domains will be denoted by $\mathcal{Z}_{\infty}^{*}$.

REMARK 3.1. Observe that the origin lies outside every domain in $\mathcal{Z}_{\infty}^{*}$. Indeed, assume that $0 \in \Omega$ for some $\Omega \in \mathcal{Z}_{\infty}^{*}$. Since $\Omega \neq \mathbb{C}$, there exists a finite point $v_{0} \in \partial \Omega$ such that $\left[0, v_{0}\right) \subset \Omega$. Hence $l^{+}[v]$ does not lie in $\Omega$ for any point $v$ of the segment $\left[0, v_{0}\right)$. 
Definition 3.2. Let $\mathcal{S}_{\infty}^{*}(\mathbb{H})$ denote the class of all univalent analytic functions $f$ in $\mathbb{H}$ such that $f(\mathbb{H}) \in \mathcal{Z}_{\infty}^{*}$. Functions belonging to $\mathcal{S}_{\infty}^{*}(\mathbb{H})$ will be called starlike w.r.t. the boundary point at infinity.

It is obvious that for every $f \in \mathcal{S}_{\infty}^{*}(\mathbb{H})$ there is some point on the boundary of $\mathbb{H}$ which "corresponds" to infinity on the boundary of $f(\mathbb{H})$. In what follows we will use a kind of boundary normalization for every $f \in \mathcal{S}_{\infty}^{*}(\mathbb{H})$ by saying that $\infty \in \partial \mathbb{H}$ corresponds to $\infty \in \partial f(\mathbb{H})$. Since, in general, we cannot extend a function $f$ to $\partial \mathbb{H}$, in order to be precise, we will apply the notion of prime ends to formulate this normalization. Below we construct a prime end $p_{\infty}(\Omega)$ for every $\Omega \in \mathcal{Z}_{\infty}^{*}$ and next using the Prime End Theorem we associate $\infty \in \partial \mathbb{H}$ with $p_{\infty}(\Omega)$.

Construction of a prime end for a domain starlike w.r.t. the boundary point at infinity. For an arbitrary domain in $\mathcal{Z}_{\infty}^{*}$ we introduce a special null chain $\left(C_{n}\right)$.

Let us recall that a crosscut of a domain $G \subset \overline{\mathbb{C}}$ is an open Jordan arc $C$ in $G$ such that $\bar{C}=C \cup\{a, b\}$, where $a, b \in \partial G$.

Let $\Omega \in \mathcal{Z}_{\infty}^{*}$. Since $\Omega \neq \mathbb{C}$, there exists a finite boundary point $v_{0}$ of $\Omega$ such that $l^{+}\left[v_{0}\right] \backslash\left\{v_{0}\right\}$ lies in $\Omega$. For each $t \in(0, \infty)$ set $C(t)=\{v \in \mathbb{C}$ : $\left.\left|v-v_{0}\right|=t\left|v_{0}\right|\right\}$. It is clear that $\Omega \cap C(t) \neq \emptyset$ for every $t \in(0, \infty)$. By [5, Proposition 2.13 , p. 28], for every fixed $t \in(0, \infty)$ there are countably many crosscuts $C_{k}(t) \subset C(t), k \in \mathbb{N}$, of $\Omega$. We denote by $\Omega_{0}(t) \subset \Omega$ the component of $\Omega \backslash C(t)$ containing the halfline $l^{+}\left[(1+t) v_{0}\right] \backslash\left\{(1+t) v_{0}\right\}$, and by $Q(t) \in \bigcup_{k \in \mathbb{N}} C_{k}(t)$ the crosscut containing the point $(1+t) v_{0}$. So $Q(t) \subset \partial \Omega_{0}(t)$. Let now $\left(t_{n}\right)$ be a strictly increasing sequence of points in $(0, \infty)$ such that $\lim _{n \rightarrow \infty} t_{n}=\infty$ and let $\left(Q\left(t_{n}\right)\right)$ be the corresponding sequence of crosscuts of $\Omega$. It is easy to observe that

(a) $\overline{Q\left(t_{n}\right)} \cap \overline{Q\left(t_{n+1}\right)}=\emptyset$ for every $n \in \mathbb{N}$.

(b) $\Omega_{0}\left(t_{n+1}\right) \subset \Omega_{0}\left(t_{n}\right)$ for every $n \in \mathbb{N}$.

(c) $\operatorname{diam}^{\#} Q\left(t_{n}\right) \rightarrow 0$ as $n \rightarrow \infty$, where $\operatorname{diam}^{\#} B$ is the spherical diameter of the set $B \subset \overline{\mathbb{C}}$.

Therefore $\left(C_{n}\right)=\left(Q\left(t_{n}\right)\right)$ forms a null chain of $\Omega$. Notice also that the null chain $\left(C_{n}\right)$ is independent of the choice of the sequence $\left(t_{n}\right)$. The equivalence class of the null chain $\left(C_{n}\right)$ defines the prime end denoted by $p_{\infty}(\Omega)$.

We can also derive that infinity is a unique principal point of the prime end $p_{\infty}(\Omega)$. Therefore, the following proposition holds ([5, p. 39]).

Proposition 3.1. For every $\Omega \in \mathcal{Z}_{\infty}^{*}$ the prime end $p_{\infty}(\Omega)$ is of the first or of the second kind.

Let $g$ be an analytic univalent mapping of $\mathbb{D}$ onto $\Omega$. Then there exists a bijective mapping $\widehat{g}$ of $\mathbb{T}$ onto the set $p(\Omega)$ of all prime ends of $\Omega([5, \mathrm{p} .30])$. 
If $h(w)=(w-1) /(w+1), w \in \overline{\mathbb{H}}$, then $\widehat{f}=\widehat{g} \circ h$ is a bijective mapping of the closed imaginary axis $\partial \mathbb{H} \cup\{\infty\}$ onto $p(\Omega)$. Thus there there is a unique $w_{0} \in \partial \mathbb{H} \cup\{\infty\}$ such that $p_{\infty}(\Omega)=\widehat{f}\left(w_{0}\right)$.

By using Proposition 3.1 and [5, Corollary 2.17, p. 35], we get

Proposition 3.2. Every function $f \in \mathcal{S}_{\infty}^{*}(\mathbb{H})$ such that $p_{\infty}(f(\mathbb{H}))=$ $\widehat{f}(\infty)$ has a radial limit

$$
\lim _{\mathbb{R} \ni x \rightarrow \infty} f(x)=\infty .
$$

To present an analytic characterization of the class $\mathcal{S}_{\infty}^{*}(\mathbb{H})$ we will need the following theorem.

Theorem 3.1. Let $f \in \mathcal{A}(\mathbb{H})$ be univalent in $\mathbb{H}$. Then $f \in \mathcal{S}_{\infty}^{*}(\mathbb{H})$ and $p_{\infty}(\mathbb{H})=\widehat{f}(\infty)$ if and only if $f\left(\mathbb{H}_{c}\right) \in \mathcal{Z}_{\infty}^{*}$ for every $c>0$.

Proof. Assume that $f \in \mathcal{S}_{\infty}^{*}(\mathbb{H})$ and $w_{0}=\infty$ corresponds to the prime end $p_{\infty}(f(\mathbb{H}))$. For each $t>1$ define

$$
q_{t}(w)=f^{-1}(t f(w)), \quad w \in \mathbb{H} .
$$

Since $f(\mathbb{H})$ is a domain starlike w.r.t. infinity, we have $t f(w) \in f(\mathbb{H})$ for every $t>1$ and $w \in \mathbb{H}$. The univalence of $f$ shows that the function $q_{t}$ is well defined for each $t>1$.

Now fix $t>1$ and let $v_{0} \in \partial f(\mathbb{H})$ be such that $l^{+}\left[v_{0}\right] \backslash\left\{v_{0}\right\} \subset f(\mathbb{H})$. Consider the sequence $\left(v_{n}\right)=\left(t^{n} v_{0}\right)$ of points in $l^{+}\left[v_{0}\right]$ and the corresponding sequence $\left(w_{n}\right)=\left(f^{-1}\left(v_{n}\right)\right)$ of points in $\mathbb{H}$. Using the same notations as before let $C\left(t_{n}\right)=\left\{v \in \mathbb{C}:\left|v-v_{0}\right|=\left(t^{n}-1\right)\left|v_{0}\right|\right\}$ and let $Q\left(t_{n}\right) \subset C\left(t_{n}\right)$ denote the crosscut of $f(\mathbb{H})$ containing the point $v_{n}$. Therefore $\left(Q\left(t_{n}\right)\right)$ is a null chain representing the prime end $p_{\infty}(f(\mathbb{H}))$. By the Prime End Theorem [5, p. 30], $\left(f^{-1}\left(Q\left(t_{n}\right)\right)\right)$ is a null chain in $\mathbb{H}$ that separates $v_{0}$ and infinity for large $n$. Since $w_{n}=f^{-1}\left(v_{n}\right) \in f^{-1}\left(Q\left(t_{n}\right)\right)$ and $\operatorname{diam}^{\#} f^{-1}\left(Q\left(t_{n}\right)\right) \rightarrow 0$ as $n \rightarrow \infty$, we conclude that $\lim _{n \rightarrow \infty} w_{n}=\infty$. Observe that

$$
q_{t}\left(w_{n}\right)=f^{-1}\left(t v_{n}\right)=w_{n+1} .
$$

Let now

$$
a_{n}=\frac{\left|q_{t}\left(w_{n}\right)+1\right|-\left|q_{t}\left(w_{n}\right)-1\right|}{\left|w_{n}+1\right|-\left|w_{n}-1\right|} \cdot \frac{\left|w_{n}+1\right|}{\left|q_{t}\left(w_{n}\right)+1\right|}, \quad n \in \mathbb{N} .
$$

Hence

$$
a_{n}=\frac{\left|w_{n+1}+1\right|-\left|w_{n+1}-1\right|}{\left|w_{n}+1\right|-\left|w_{n}-1\right|} \cdot \frac{\left|w_{n}+1\right|}{\left|w_{n+1}+1\right|}
$$

for all $n \in \mathbb{N}$. Consequently, 


$$
\begin{aligned}
\lim _{n \rightarrow \infty}\left(a_{1} a_{2} \ldots a_{n}\right) & =\lim _{n \rightarrow \infty}\left\{\frac{\left|w_{n+1}+1\right|-\left|w_{n+1}-1\right|}{\left|w_{1}+1\right|-\left|w_{1}-1\right|} \cdot \frac{\left|w_{1}+1\right|}{\left|w_{n+1}+1\right|}\right\} \\
& =\lim _{n \rightarrow \infty}\left\{\frac{\left|w_{1}+1\right|}{\left|w_{1}+1\right|-\left|w_{1}-1\right|}\left(1-\frac{1}{\left|1+\frac{1}{w_{n+1}}\right|}\right)\right\}=0 .
\end{aligned}
$$

Hence infinitely many $a_{n}$ must satisfy $0<a_{n} \leq 1$, so there exists a convergent subsequence $\left(a_{n_{k}}\right)$ such that

$$
0 \leq \lim _{k \rightarrow \infty} a_{n_{k}}=\alpha(t) \leq 1
$$

which means that

$$
\lim _{k \rightarrow \infty}\left\{\frac{\left|q_{t}\left(w_{n_{k}}\right)+1\right|-\left|q_{t}\left(w_{n_{k}}\right)-1\right|}{\left|w_{n_{k}}+1\right|-\left|w_{n_{k}}-1\right|} \cdot \frac{\left|w_{n_{k}}+1\right|}{\left|q_{t}\left(w_{n_{k}}\right)+1\right|}\right\}=\alpha(t) \leq 1
$$

for every fixed $t>1$. In fact, in view of Remark 2.2, $\alpha(t)>0$ for every $t>1$.

Hence $q_{t}$ satisfies the assumptions of Lemma 2.2 , and since $\alpha(t) \leq 1$ for every $t>1$, we derive that

$$
q_{t}\left(\mathbb{H}_{c}\right) \subset \mathbb{H}_{c / \alpha(t)} \subset \mathbb{H}_{c}
$$

for every $c>0$. This yields $t f\left(\mathbb{H}_{c}\right) \subset f\left(\mathbb{H}_{c}\right)$ for every $t>1$. Therefore $f\left(\mathbb{H}_{c}\right) \in \mathcal{Z}_{\infty}^{*}$ for every $c>0$. Conversely, assume that $f\left(\mathbb{H}_{c}\right) \in \mathcal{Z}_{\infty}^{*}$ for every $c>0$. Since $\infty \in \partial f\left(\mathbb{H}_{c}\right)$ for every $c>0$ and

$$
f(\mathbb{H})=\bigcup_{c>0} f\left(\mathbb{H}_{c}\right),
$$

it follows that $\infty \in \partial f(\mathbb{H})$ and $f(\mathbb{H}) \in \mathcal{Z}_{\infty}^{*}$. Observe also that there exists a prime end $p_{\infty}(f(\mathbb{H}))$ which corresponds to a point $w_{0} \in \partial \mathbb{H} \cup\{\infty\}$. We need to show that $w_{0}=\infty$.

To this end, fix $c>0$ and suppose that $w_{0} \neq \infty$. Let $v_{0} \in f(\mathbb{H})$ be such that $l^{+}\left[v_{0}\right] \backslash\left\{v_{0}\right\} \subset f(\mathbb{H})$.

Let $C(t)=\left\{v \in \mathbb{C}:\left|v-v_{0}\right|=t\left|v_{0}\right|\right\}, t>0$. Repeating the construction of a null chain for the domain $f(\mathbb{H})$ and using the same notation let $Q(t), t>0$, denote the crosscut of $f(\mathbb{H})$ containing the point $(1+t) v_{0}$. Choosing a strictly increasing sequence $\left(t_{n}\right)$ of points in $(1, \infty)$ such that $\lim _{n \rightarrow \infty} t_{n}=\infty$ let $\left(Q\left(t_{n}\right)\right)$ be the corresponding sequence of crosscuts of $f(\mathbb{H})$ which represents the prime end $p_{\infty}(f(\mathbb{H}))$ corresponding in a unique way to $w_{0} \in \partial \mathbb{H} \cup\{\infty\}$. By the Prime End Theorem, $\left(f^{-1}\left(Q\left(t_{n}\right)\right)\right)$ is a null chain that separates in $\mathbb{H}$ the points $w_{0}$ and $v_{0}$ for large $n$. Since $v_{0} \neq \infty$ and $\operatorname{diam}^{\#} f^{-1}\left(Q\left(t_{n}\right)\right) \rightarrow 0$ as $n \rightarrow \infty$ we see that

$$
f^{-1}\left(Q\left(t_{n}\right)\right) \cap \mathbb{H}_{c}=\emptyset
$$

for large $n$. 
On the other hand, $f\left(\mathbb{H}_{c}\right)$ is in $\mathcal{Z}_{\infty}^{*}$, which implies that $Q\left(t_{n}\right) \cap f\left(\mathbb{H}_{c}\right) \neq \emptyset$ for large $n \in \mathbb{N}$. This contradicts (3.1) and finally proves that $w_{0}=\infty$, so $p_{\infty}(f(\mathbb{H}))=\widehat{f}(\infty)$. The proof of the theorem is finished.

In the proof of the next theorem we need the lemma below.

Lemma 3.1. If $f \in \mathcal{A}(\mathbb{H})$ is univalent in $\mathbb{H}$ and $f(w) \neq 0$ for $w \in \mathbb{H}$, then

$$
\left|\frac{f^{\prime}(x)}{f(x)}\right| \leq \frac{2}{x}, \quad x>0
$$

Proof. Since $f \in \mathcal{A}(\mathbb{H})$ is univalent in $\mathbb{H}$, the function

$$
g(z)=f\left(\frac{1+z}{1-z}\right)=f(w), \quad z \in \mathbb{D},
$$

where $w=(1+z) /(1-z)$, is analytic univalent in $\mathbb{D}$ and maps it onto the domain $\Omega=f(\mathbb{H})$. Hence

$$
\frac{\left|g^{\prime}(z)\right|}{\operatorname{dist}(g(z), \partial g(\mathbb{D}))} \leq \frac{4}{1-|z|^{2}}
$$

for $z \in \mathbb{D}$ (see $[5$, p. 92]). For any $v \notin \Omega$, the above shows that

$$
\left|\frac{g^{\prime}(z)}{g(z)-v}\right| \leq \frac{4}{1-|z|^{2}}
$$

for $z \in \mathbb{D}$. Hence

$$
\left|\frac{f^{\prime}(w)}{f(w)-v}\right| \leq \frac{8}{|w+1|^{2}-|w-1|^{2}}
$$

for $w \in \mathbb{H}$. Therefore for $v=0$ and $w=x>0$ we obtain (3.2).

To present an analytic characterization of the class $\mathcal{S}_{\infty}^{*}(\mathbb{H})$ it is useful to define the following class of functions.

Definition 3.3. For $\alpha>0$ let $\mathcal{P}(\alpha ; \mathbb{H})$ be the subclass of all functions $q \in \mathcal{P}(\mathbb{H})$ satisfying $(2.4)$ and (2.5).

TheOREM 3.2. If $f \in \mathcal{S}_{\infty}^{*}(\mathbb{H})$ and $p_{\infty}(f(\mathbb{H}))=\widehat{f}(\infty)$, then there exists $\alpha \in(0,1]$ and a function $q \in \mathcal{P}(\alpha ; \mathbb{H})$ such that

$$
\frac{f^{\prime}(w)}{f(w)}=\frac{2}{q(w)}, \quad w \in \mathbb{H} .
$$

Proof. Define

$$
q(w)=2 \frac{f(w)}{f^{\prime}(w)}, \quad w \in \mathbb{H} .
$$

Since $f^{\prime}(w) \neq 0$ in $\mathbb{H}, q$ is analytic in $\mathbb{H}$.

We will prove that $q$ is in $\mathcal{P}(\alpha ; \mathbb{H})$ for some $\alpha \in(0,1]$. 
1. First we show that $\operatorname{Re} q(w)>0$ for $w \in \mathbb{H}$, i.e.

$$
\operatorname{Re}\left\{\frac{f^{\prime}(w)}{f(w)}\right\}>0, \quad w \in \mathbb{H} .
$$

Remark 3.1 yields $0 \notin f(\mathbb{H})$. Therefore the function

$$
\gamma_{c}=\partial \mathbb{H}_{c} \ni w \mapsto \arg f(w)
$$

is well defined on the analytic arc $\gamma_{c}$. We use the following parametrization:

$$
\gamma_{c}: w=\gamma(y)=c+i y, \quad y \in \mathbb{R} .
$$

From Theorem 3.1 it follows that $f\left(\mathbb{H}_{c}\right) \in \mathcal{Z}_{\infty}^{*}$ for every $c>0$. This means geometrically that the function

$$
\mathbb{R} \ni y \mapsto \arg f(\gamma(y))
$$

is monotonic on the analytic arc $\gamma_{c}$ for every $c>0$. Since $f$ is a conformal mapping, it preserves the orientation of $\gamma_{c}$. We have

$$
\begin{aligned}
\frac{d}{d y} \arg f(\gamma(y)) & =\frac{d}{d y} \operatorname{Im} \log f(\gamma(y))=\operatorname{Im}\left\{\gamma^{\prime}(y) \frac{f^{\prime}(\gamma(y))}{f(\gamma(y))}\right\} \\
& =\operatorname{Re}\left\{\frac{f^{\prime}(\gamma(y))}{f(\gamma(y))}\right\} \geq 0
\end{aligned}
$$

for $y \in \mathbb{R}$. By the above

$$
\operatorname{Re}\left\{\frac{f^{\prime}(w)}{f(w)}\right\} \geq 0
$$

for $w \in \mathbb{H}$.

Assume now that equality holds in (3.6) for some $w_{0} \in \mathbb{H}$. By the maximum principle for harmonic functions it holds in the whole halfplane $\mathbb{H}$, which implies that there exists $b \in \mathbb{R} \backslash\{0\}$ so that

$$
\frac{f^{\prime}(w)}{f(w)} \equiv b i, \quad w \in \mathbb{H} .
$$

But the solution

$$
f(w)=f_{0}(w)=a \exp (i b w), \quad w \in \mathbb{H}, a \in \mathbb{C} \backslash\{0\},
$$

of the last equation is not univalent in $\mathbb{H}$. So $f_{0} \notin \mathcal{S}_{\infty}^{*}(\mathbb{H})$ and hence the strict inequality holds in (3.6).

2. Now we prove that the function $q$ satisfies the conditions (2.11) and (2.12) of Corollary 2.1. Let $\left(x_{n}\right)$ be an arbitrary sequence of positive real numbers such that $\lim _{n \rightarrow \infty} x_{n}=\infty$. Since, by Remark 3.1, $0 \notin f(\mathbb{H})$, applying Lemma 3.1 we have

$$
\left|q\left(x_{n}\right)\right|=2\left|\frac{f\left(x_{n}\right)}{f^{\prime}\left(x_{n}\right)}\right| \geq x_{n},
$$


which implies that $\lim _{n \rightarrow \infty} q\left(x_{n}\right)=\infty$. Moreover Lemma 3.1 yields

$$
\frac{x_{n}}{\left|q\left(x_{n}\right)\right|}=\frac{x_{n}}{2}\left|\frac{f^{\prime}\left(x_{n}\right)}{f\left(x_{n}\right)}\right| \leq 1
$$

for $n \in \mathbb{N}$. Take a convergent subsequence $\left(x_{n_{k}}\right)$ such that

$$
\lim _{k \rightarrow \infty} \frac{x_{n_{k}}}{\left|q\left(x_{n_{k}}\right)\right|}=\alpha_{1} \leq 1
$$

Hence

$$
\lim _{k \rightarrow \infty} \frac{x_{n_{k}}+1}{\left|q\left(x_{n_{k}}\right)+1\right|}=\lim _{k \rightarrow \infty} \frac{x_{n_{k}}\left(1+\frac{1}{x_{n_{k}}}\right)}{\left|q\left(x_{n_{k}}\right)\right| 1+\frac{1}{q\left(x_{n_{k}}\right)} \mid}=\alpha_{1} .
$$

It is easy to check that for $x>0$,

$$
|q(x)+1|-|q(x)-1| \leq 2 .
$$

In particular, the last inequality holds for $x=x_{n_{k}}$ so there exists a subsequence $\left(x_{n_{k_{l}}}\right)$ such that

$$
\lim _{l \rightarrow \infty}\left\{\left|q\left(x_{n_{k_{l}}}\right)+1\right|-\left|q\left(x_{n_{k_{l}}}\right)-1\right|\right\}=2 \alpha_{2} \leq 2 .
$$

Consequently,

$$
\lim _{l \rightarrow \infty}\left\{\left(\left|q\left(x_{n_{k_{l}}}\right)+1\right|-\left|q\left(x_{n_{k_{l}}}\right)-1\right|\right) \frac{x_{n_{k_{l}}}+1}{\left|q\left(x_{n_{k_{l}}}\right)+1\right|}\right\}=2 \alpha_{1} \alpha_{2}=2 \alpha,
$$

where $\alpha \in[0,1]$. By Remark 2.2, $\alpha \in(0,1]$. In this way the function $q$ satisfies the assumptions of Corollary 2.1.

This ends the proof of the theorem.

Theorem 3.3. Let $f \in \mathcal{A}(\mathbb{H})$. Assume that there exists an $\alpha \in(0,1]$ and a function $q \in \mathcal{P}(\mathbb{H})$ with $\lim _{w \rightarrow \infty} q(w)=\infty$ such that

$$
\lim _{w \rightarrow \infty} \frac{q(w)}{w}=\frac{1}{\alpha} .
$$

If

$$
\frac{f^{\prime}(w)}{f(w)}=\frac{2}{q(w)}, \quad w \in \mathbb{H},
$$

then $f \in \mathcal{S}_{\infty}^{*}(\mathbb{H})$ and $p_{\infty}(f(\mathbb{H}))=\widehat{f}(\infty)$.

Proof. Notice that from (3.8) it follows that $f$ is locally univalent in $\mathbb{H}$. Also $f(w) \neq 0$ for $w \in \mathbb{H}$.

Now we prove that $f$ is univalent in $\mathbb{H}$.

Consider again the function (3.3) defined on the analytic arcs $\gamma_{c}=\partial \mathbb{H}_{c}$ for each $c>0$, parametrized by (3.4). Repeating the calculations (3.5) we 
see that the condition (3.8) implies

$$
\frac{d}{d y} \arg f(\gamma(y))>0, \quad y \in \mathbb{R},
$$

i.e. the function (3.3) is strictly increasing on each $\gamma_{c}$. By monotonicity the limits

$$
\psi_{c}=\lim _{y \rightarrow+\infty} \arg f(\gamma(y)), \quad \varphi_{c}=\lim _{y \rightarrow-\infty} \arg f(\gamma(y))
$$

(possibly infinite) both exist and $\psi_{c}-\varphi_{c}>0$ (see [4, Theorem 1, p. 17]). We will prove that $\psi_{c}-\varphi_{c} \leq 2 \pi$ for every $c>0$.

For every $c>0$ and $R>c$ consider two $\operatorname{arcs}$ in $\mathbb{H}$ :

$$
\begin{aligned}
& \gamma_{c, R}=\left\{c+i y: y \in\left[-\sqrt{R^{2}-c^{2}}, \sqrt{R^{2}-c^{2}}\right]\right\}, \\
& \sigma_{c, R}=\{w \in \mathbb{C}:|w|=R\} \cap \overline{\mathbb{H}}_{c} .
\end{aligned}
$$

We will use the following parametrization:

$$
\sigma_{c, R}: w=\sigma(t)=R e^{i t}, \quad t \in[-t(c, R), t(c, R)],
$$

where $t(c, R)=\arctan \left(\sqrt{R^{2}-c^{2}} / c\right)$. The arc $\gamma_{c, R}$ will be parametrized by (3.3) where $\left.y \in\left[-\sqrt{R^{2}-c^{2}}, \sqrt{R^{2}-c^{2}}\right]\right\}$.

(a) Assume first that $\alpha \in(0,1)$. Fix $c>0$. Then by (3.7) there exists $R_{1}>c$ such that $|w / q(w)| \leq \alpha<1$ for $w \in \mathbb{H} \backslash \mathbb{D}\left(0, R_{1}\right)$. Take $R>R_{1}$. Hence $\mathbb{H} \backslash \mathbb{D}(0, R) \subset \mathbb{H} \backslash \mathbb{D}\left(0, R_{1}\right)$.

Since $\gamma_{c, R} \cup \sigma_{c, R}$ is a closed curve lying in $\mathbb{H}$, we have

$$
\begin{aligned}
& \arg f\left(\gamma\left(\sqrt{R^{2}-c^{2}}\right)\right)-\arg f\left(\gamma\left(-\sqrt{R^{2}-c^{2}}\right)\right)=\Delta_{\gamma_{c, R}} \arg f(w) \\
& \quad=\operatorname{Im} \int_{\gamma_{c, R}} \frac{f^{\prime}(w)}{f(w)} d w=\operatorname{Im} \int_{\gamma_{c, R}} \frac{2}{q(w)} d w=\operatorname{Im} \int_{\sigma_{c, R}} \frac{2}{q(w)} d w \\
& \quad=\operatorname{Im} \int_{-t(c, R)}^{t(c, R)} \frac{2 i R e^{i t}}{q\left(R e^{i t}\right)} d t \leq 2 \int_{-t(c, R)}^{t(c, R)} \frac{R}{\left|q\left(R e^{i t}\right)\right|} d t \\
& \quad \leq 4 t(c, R) \alpha \leq 2 \pi \alpha<2 \pi .
\end{aligned}
$$

If now $R \rightarrow \infty$, then $t(c, R) \rightarrow \pi / 2$, which implies

$$
\begin{aligned}
0 & <\psi_{c}-\varphi_{c} \\
& =\lim _{R \rightarrow \infty}\left(\arg f\left(\sqrt{R^{2}-c^{2}}\right)-\arg f\left(-\sqrt{R^{2}-c^{2}}\right)\right) \leq 2 \pi .
\end{aligned}
$$

This means that $f\left(\mathbb{H}_{c}\right) \subset V_{c}$, where $V_{c}$ is the sector with vertex at the origin and with opening angle $\psi_{c}-\varphi_{c}$. Since $f\left(\mathbb{H}_{c_{2}}\right) \subset f\left(\mathbb{H}_{c_{1}}\right)$ for $0<c_{1}<$ $c_{2}$, we have $V_{c_{2}} \subset V_{c_{1}}$. Hence $V=\bigcup_{c>0} V_{c}$ is a sector with vertex at the origin and with opening angle $\psi-\varphi=\sup \left\{\psi_{c}-\varphi_{c}: c>0\right\}$. Obviously, 
$0<\psi-\varphi \leq 2 \pi$. As

$$
f(\mathbb{H})=\bigcup_{c>0} f\left(\mathbb{H}_{c}\right) \subset \bigcup_{c>0} V_{c}=V,
$$

there exists $\varphi_{0} \in \mathbb{R}$ such that

$$
\begin{aligned}
\varphi_{0}<\arg f(w) & =\operatorname{Im} \log f(w)=\operatorname{Im} g(w) \\
<\varphi_{0}+\psi-\varphi & \leq \varphi_{0}+2 \pi, \quad w \in \mathbb{H},
\end{aligned}
$$

where $g(w)=\log f(w), w \in \mathbb{H}$, is well defined in $\mathbb{H}$. But (3.8) yields

$$
\operatorname{Re}\left\{\frac{f^{\prime}(w)}{f(w)}\right\}=\operatorname{Re}\left\{g^{\prime}(w)\right\}>0, \quad w \in \mathbb{H} .
$$

Now $[5$, Proposition 1.10, p. 16] yields the univalence of $g$ in $\mathbb{H}$. Since $f(w)=$ $\exp (g(w)), w \in \mathbb{H}$, using (3.10) we deduce finally that $f$ is univalent in $\mathbb{H}$.

(b) Let now $\alpha=1$. Fix $c>0$. For $n \in \mathbb{N}$ there exists $R_{1}(n)>c$ such that $|w / q(w)| \leq 1+1 / n$ for $w \in \mathbb{H} \backslash \mathbb{D}\left(0, R_{1}(n)\right)$. For each $n \in$ $\mathbb{N}$ take $R(n)>R_{1}(n)$ in such a way that $\lim _{n \rightarrow \infty} R(n)=\infty$. Clearly, $\mathbb{H} \backslash \mathbb{D}(0, R(n)) \subset \mathbb{H} \backslash \mathbb{D}\left(0, R_{1}(n)\right)$. Let $\gamma_{c, R(n)}$ and $\sigma_{c, R(n)}$ be defined as in part (a). Since $\gamma_{c, R(n)} \cup \sigma_{c, R(n)}$ is a closed curve lying in $\mathbb{H}$, we have, as in (3.9), for each $n \in \mathbb{N}$,

$$
\begin{aligned}
\Delta_{\gamma_{c, R(n)}} \arg f(w) & \leq 2 \int_{-t(c, R(n))}^{t(c, R(n))}\left|\frac{R(n) e^{i t}}{q\left(R(n) e^{i t}\right)}\right| d t \\
& \leq 4\left(1+\frac{1}{n}\right) t(c, R(n)) \alpha \leq 2\left(1+\frac{1}{n}\right) \pi,
\end{aligned}
$$

and we complete the proof as before.

ExAmples. Now we present some examples of functions. The first two examples are geometrically obvious. The first function maps univalently $\mathbb{H}$ onto a slit plane, the second one maps $\mathbb{H}$ onto a wedge. The third example does not exhibit an evident geometrical property. Therefore Theorem 3.3 offers a useful analytical method to check if the function is an element of $\mathcal{S}_{\infty}^{*}(\mathbb{H})$.

1. $f(w)=\sqrt{w^{2}+a^{2}}, w \in \mathbb{H}, a \geq 0$. Then

$$
q(w)=2 \frac{f(w)}{f^{\prime}(w)}=2 \frac{w^{2}+a^{2}}{w}=2\left(w+\frac{a^{2}}{w}\right), \quad w \in \mathbb{H} .
$$

Consequently, $q \in \mathcal{A}(\mathbb{H})$, $\operatorname{Re} q(w)>0$ for $w \in \mathbb{H}$, and $\lim _{w \rightarrow \infty} q(w) / w=2$ so (3.7) is satisfied with $\alpha=1 / 2$. By Theorem 3.3, $f \in \mathcal{S}_{\infty}^{*}(\mathbb{H})$.

2. $f(w)=w^{\mu}, w \in \mathbb{H}, \mu \in(0,2]$. Then

$$
q(w)=\frac{2}{\mu} w, \quad w \in \mathbb{H} .
$$


Consequently, $q \in \mathcal{A}(\mathbb{H}), \operatorname{Re} q(w)>0$ for $w \in \mathbb{H}$, and $\lim _{w \rightarrow \infty} q(w) / w=2 / \mu$ so (3.7) is satisfied with $\alpha=\mu / 2$. By Theorem 3.3, $f \in \mathcal{S}_{\infty}^{*}(\mathbb{H})$ for each $\mu \in(0,2]$.

3. $f(w)=(w+1)^{\beta} \exp \left(\frac{1-w}{1+w}\right), w \in \mathbb{H}, \beta>0$. Then

$$
q(w)=2 \frac{(w+1)^{2}}{\beta w+\beta-2}, \quad w \in \mathbb{H} .
$$

For $\beta \in(0,2)$ the function $q$ has a pole at $w=(2-\beta) / \beta$ so $q \notin \mathcal{A}(\mathbb{H})$.

For $\beta \geq 2, q \in \mathcal{A}(\mathbb{H})$. Moreover, for $w \in \mathbb{H}$, i.e. for $w=x+i y$ with $x>0$, we have

$$
\operatorname{Re} q(w)=2 \frac{(x+1)^{2}(\beta x+\beta-2)+(2+\beta(x+1)) y^{2}}{(\beta(x+1)-2)^{2}+\beta^{2} y^{2}}>0 .
$$

Since $\lim _{w \rightarrow \infty} q(w) / w=2 / \beta$, we conclude that (3.7) is satisfied only for $\beta=2$, i.e. for $\alpha=1$. By Theorem 3.3, $f \in \mathcal{S}_{\infty}^{*}(\mathbb{H})$ for $\beta=2$.

\section{References}

[1] C. Carathéodory, Conformal Representation, Cambridge Univ. Press, Cambridge, 1963.

[2] G. Dimkov, J. Stankiewicz and Z. Stankiewicz, On a class of starlike functions defined in a halfplane, Ann. Polon. Math. 55 (1991), 81-86.

[3] G. Julia, Extension nouvelle d'un lemme de Schwarz, Acta Math. 42 (1918), 349-355.

[4] S. Łojasiewicz, Introduction to the Theory of Real Functions, PWN, Warszawa, 1976 (in Polish).

[5] C. Pommerenke, Boundary Behaviour of Conformal Maps, Springer, Berlin, 1992.

[6] J. Stankiewicz and Z. Stankiewicz, On the class of functions regular in a halfplane, Bull. Polish Acad. Sci. Math. 39 (1991), 49-56.

Department of Mathematics

Technical University of Rzeszów

W. Pola 2

35-959 Rzeszów, Poland

E-mail: alecko@prz.rzeszow.pl 